D0I: 10.12957/demetra.2015.15404

\title{
Fatores associados à retenção de peso pós-parto de mulheres atendidas no setor de vacinação da Policlínica Municipal de Viçosa-MG
}

\section{Factors associated with postpartum weight retention in women served in the vaccination sector of the Municipal Clinic in the city of Viçosa-MG, Brazil}

Patrícia Pereira de Almeida'

Raquel Maria Amaral Araújo?

1 Universidade Federal de Viçosa, Departamento de Nutrição e Saúde. Viçosa-MG, Brasil.

Correspondência / Correspondence

Patrícia Pereira de Almeida

E-mail: patricialmeida10@gmail.com

\section{Resumo}

Objetivos: O objetivo deste estudo foi avaliar a retenção de peso de mulheres do $2^{\circ}$ ao $18^{\circ}$ mês pós-parto atendidas no serviço de imunização do município de Viçosa-MG, quanto aos fatores socioeconômicos, comportamentais, obstétricos, antropométricos e da ocorrência do aleitamento materno neste período. Métodos: Trata-se de estudo transversal realizado com mulheres do $2^{\circ}$ ao $18^{\circ}$ mês pós-parto. Foi aplicado um questionário semiestruturado contendo itens relativos a identificação, antropometria, condições sociodemográficas, amamentação e hábitos gerais. Houve autorrelato do peso pré-gestacional e do peso ganho na gestação, e aferição do peso e altura das mães, sendo que a retenção de peso foi calculada pela diferença entre peso atual e peso prégestacional. Foi realizada estatística descritiva, através de medidas de tendência central e de dispersão. Resultados: Verificou-se elevada prevalência de sobrepeso no período menor ou igual a seis meses pós-parto e de sobrepeso e obesidade no período maior que seis meses pós-parto, sendo que este último achado representa uma preocupação. Observou-se também maior retenção de peso entre mulheres que apresentaram ganho de peso excessivo durante a gestação. Conclusão: Embora o pequeno número amostral tenha limitado a realização de algumas associações estatísticas, observa-se relação entre retenção de peso pós-parto e ganho de peso gestacional. Como ponto positivo, pode ser mencionada a elevada prevalência de aleitamento materno entre as mães estudadas.

Palavras-chave: Obesidade. Período Pós-Parto. Ganho de Peso. Índice de Massa Corporal. 


\section{Abstract}

Objective: This study aimed to evaluate the weight retention of women from the 2nd to the 18th month postpartum served in the vaccination sector of the Municipal Polyclinic in ViçosaMG, Brazil, concerning socioeconomic, behavioral, obstetrical, anthropometric factors and the occurrence of breastfeeding in this period. Methods: This was a cross-sectional study of women from the 2nd to the 18th month postpartum. A semi-structured questionnaire containing items related to identification, anthropometry, sociodemographic conditions, breastfeeding and general habits of the participants was applied. There were selfreport of pregnancy weight and weight gain during pregnancy, and measurement of height and weight of mothers; weight retention was calculated as the difference between current and pre-pregnancy weight. Descriptive statistics were performed by measures of central tendency and dispersion. Results: There was high prevalence of overweight in less than or equal to six months postpartum and overweight and obesity in the period longer than six months postpartum; the latter finding is a concern. We also observed greater weight retention among women with excessive weight gain during pregnancy. Conclusions: Although the small sample size limited the fulfillment of some statistical associations, there was relationship between retention of gestational weight gain and postpartum weight. On the positive side, it can be mentioned the high prevalence of breastfeeding among mothers studied.

Key words: Obesity. Postpartum Period. Weight Gain. Body Mass Index.

\section{Introdução}

A obesidade é decorrente de um conjunto de fatores genéticos e ambientais e configura um problema de saúde pública emergente em diversos grupos etários e regiões do Brasil, sendo mais prevalente em mulheres, principalmente. ${ }^{1,2}$ Segundo dados da Pesquisa de Orçamentos Familiares (2008-2009), sobrepeso e obesidade estiveram presentes em aproximadamente metade dos homens e mulheres adultos, sendo que a obesidade foi diagnosticada em 12,5\% dos homens e em 16,9\% das mulheres. Portanto, o sobrepeso e a obesidade, não só em adultos mas em todas as faixas etárias, são fatores preocupantes no âmbito da saúde brasileira. ${ }^{3}$ 
Considerando que a mulher em idade reprodutiva, sobretudo nos períodos da gestação e pós-parto, está mais vulnerável a condições que potencializam o ganho ponderal que podem levar ao estabelecimento da obesidade com o passar dos anos, é de suma importância realizar o controle de peso gestacional, a fim de promover ganho de peso adequado, prevenindo a retenção de peso pós-parto. ${ }^{1,4}$ Neste sentido, o acompanhamento adequado da gestante durante o pré-natal é fundamental para o bom prognóstico materno-fetal, bem como o controle do ganho de peso gestacional. ${ }^{2,5}$

No entanto, ressalta-se que, por vezes, as características sociais e demográficas das gestantes e nutrizes, como baixa renda, podem dificultar o acesso à assistência no pré-natal e puerpério, possibilitando o comprometimento do prognóstico da gestação e pós-parto. ${ }^{5}$ Tal fato pode ser verificado com os resultados da Pesquisa Nacional de Demografia e Saúde da Criança e da Mulher - PNDS (2006), segundo a qual 77\% das mães realizaram no mínimo seis consultas de pré-natal, porém apenas 39\% das puérperas realizaram uma consulta pós-parto, o que evidencia a negligência da assistência a mulheres nesta fase, no país. ${ }^{6}$

Vários fatores intrínsecos ao ser humano, tais como genética, gênero e idade, determinam o peso corporal; há, no entanto, fatores passíveis de modificação, como inatividade física e hábitos alimentares inadequados, que podem estar relacionados à retenção de peso pós-parto. ${ }^{2}$ Esta pode ocorrer em conjunto com o ganho de peso excessivo na gravidez, determinada por complexas interrelações, constituindo uma preocupação de saúde pública, visto que tais fatores podem predizer aumento da prevalência da obesidade. ${ }^{1,5}$

A realização do presente trabalho é justificada pelas condições propícias ao ganho de peso às quais a mãe está sujeita no pós-parto, que, associadas ao estado nutricional pré-gestacional e às modificações fisiológicas ocorridas durante a gestação, favorecem a retenção de peso. Além disso, no Brasil alguns estudos ${ }^{4,7-9}$ abordam a retenção de peso pós-parto, mais ainda é necessária a realização de mais pesquisas que atentem para o estado nutricional da mulher nessas fases, a fim de evitar o peso excessivo.

Diante do exposto, o objetivo deste estudo foi avaliar a retenção de peso de mulheres do $2^{\circ}$ ao $18^{\circ}$ mês pós-parto atendidas no setor de vacinação da Policlínica Municipal de Viçosa-MG, quanto aos fatores socioeconômicos, comportamentais, obstétricos, antropométricos e da ocorrência do aleitamento materno neste período. 


\section{Metodologia}

O estudo é do tipo transversal, foi realizado no serviço de imunização do município de ViçosaMG, com mães entre $2^{\circ}$ e o $18^{\circ}$ mês pós-parto, no período de 14 de abril a 06 de junho de 2014 . A amostra foi do tipo não probabilística por conveniência, uma vez que as mães foram convidadas a participar do estudo ao chegarem no local da coleta.

O critério de inclusão da amostra foi estar no período de 2-18 meses pós-parto, uma vez que esta é a faixa etária das crianças que mais frequentam a unidade. Os critérios de exclusão foram: ser fumante, estar em tratamento de hipertensão ou com problemas na glândula tireoide tanto no período gestacional quanto no pós-parto, ser adolescente e mulheres que não concordaram em participar da pesquisa.

Destaca-se que, segundo esses critérios de exclusão, duas adolescentes, três hipertensas e uma mulher com problema na glândula tireoide foram excluídas do estudo. Além disso, 14 mulheres possuíam uma ou mais das seguintes características: intercorrência gestacional, parto pré-termo e ser tabagista.

Os dados foram coletados por meio da aplicação de um questionário previamente elaborado pelos pesquisadores contendo itens relativos a identificação, antropometria, condições sociodemográficas, amamentação e hábitos gerais.

Para a análise da situação socioeconômica, foi utilizado o Critério de Classificação Econômica Brasil da Associação Brasileira de Empresas de Pesquisa (ABEP), que possui um questionário sobre posse de itens e grau de instrução do chefe da família, utilizando pontos de cortes próprios para a categorização nas classes econômicas de A a E. As rendas médias brutas familiares mensais eram de $\mathrm{R} \$ 9.263,00$ para a classe $\mathrm{A} ; \mathrm{R} \$ 5.241,00$ para a classe $\mathrm{B} 1 ; \mathrm{R} \$ 2.654,00$ para a classe $\mathrm{B} 2$; $\mathrm{R} \$ 1.685,00$ para a classe $\mathrm{C} 1$; $\mathrm{R} \$ 1.147,00$ para a classe C2; e $\mathrm{R} \$ 776,00$ para as classes $\mathrm{D}$ e E.

Dada a amplitude das idades pós-parto analisadas, estas foram distribuídas em duas classes: a) menor ou igual a seis meses e b) maior que seis meses. Por sua vez, o peso da criança ao nascer foi classificado como baixo para crianças nascidas com menos de $2.500 \mathrm{~g}$, peso insuficiente com 2.500 a $3.000 \mathrm{~g}$, peso normal com 3000 a $4.000 \mathrm{~g}$ e macrossomia com mais $4.000 \mathrm{~g}$ ao nascer, conforme os pontos de corte adotados pela Organização Mundial da Saúde..$^{10}$

A aferição da estatura e do peso das participantes foi realizada por meio de uma balança digital da marca BALMAK, que continha régua antropométrica medindo até dois metros e graduada a cada 0,5 centímetro, com capacidade para $200 \mathrm{Kg}$ e intervalos de 100 gramas. As medidas foram realizadas conforme os procedimentos estabelecidos pela World Health Organization (WHO). ${ }^{11}$ 
O peso pré-gestacional e o ganho de peso gestacional foram autorrelatados, já que a maioria das mães não estava com o cartão de gestante no ato da aplicação do questionário, sendo que aquelas que não se lembraram dos valores em questão foram contatadas por telefone, posteriormente à aplicação do questionário.

Em relação às variáveis antropométricas, tanto o Índice de Massa Corporal (IMC) prégestacional como o IMC do pós-parto foram classificados conforme os pontos de corte da WHO, ${ }^{12}$ que considera os IMC: < 18,5 kg/m² para baixo-peso grau 1, 18,5 a 24,99 kg/ $\mathrm{m}^{2}$ para eutrofia, $\geq$ $25,0 \mathrm{~kg} / \mathrm{m}^{2}$ para sobrepeso, $25-29,99 \mathrm{~kg} / \mathrm{m}^{2}$ para pré-obeso, $30-34,99 \mathrm{~kg} / \mathrm{m}^{2}$ para obesidade grau $1,35-39,99 \mathrm{~kg} / \mathrm{m}^{2}$ para obesidade grau 2 e $\geq 40 \mathrm{~kg} / \mathrm{m}^{2}$ para obesidade grau 3 .

O ganho ponderal ao final da gestação foi avaliado segundo as recomendações do Institute of Medicine / $\mathrm{IOM}^{13}$ para o ganho de peso total durante a gravidez de acordo com o IMC prégestacional, sendo considerados adequados ganho ponderal de 12,5-18,0 kg; 11,5-16 kg; 7-11,5 kg e 5-9 kg, para gestantes de baixo peso, eutróficas, com sobrepeso e obesas, respectivamente nesta ordem; portanto, o ganho de peso foi classificado em adequado, insuficiente ou excessivo.

A retenção de peso foi calculada pela diferença entre o peso atual e o peso pré-gestacional, ${ }^{4}$ e foi avaliada conforme a idade pós-parto, sendo mulheres com idade menor ou igual a seis meses ou maior que seis meses. Vale ressaltar que a condição para ser classificada como "não reteve peso" foi apresentar retenção de peso igual a zero ou valor negativo, representando mulheres que atualmente possuem um peso inferior ao peso pré-gestacional.

O banco de dados foi criado utilizando-se o software Microsoft Office Excel 2013 e a análise estatística realizada com o auxílio do programa Statistical Package for Social Science (SPSS) versão 20.0. Os dados foram analisados através de estatística descritiva, sendo realizadas medidas de tendência central e de dispersão.

Além disso, foi realizado o teste de Kolmogorov-Smirnov para verificar a normalidade da variável quantitativa retenção de peso, e os testes $t$ Student e análise de variância (ANOVA) com nível de significância de 5\%, a fim de comparar as médias de retenção de peso de acordo com algumas variáveis socioeconômicas, comportamentais e obstétricas. Também realizou-se o teste de Tukey, a fim de avaliar a magnitude das diferenças das médias de retenção de peso entre os grupos.

Esta pesquisa faz parte de um estudo maior sobre a mudança de comportamento frente à amamentação em gestantes e nutrizes, empregando teorias comportamentais, aprovado pelo Comitê de Ética em Pesquisa com Seres Humanos da Universidade Federal de Viçosa, sob o n 412.814 (CAAE: 16549413.2.0000.5153). Após o esclarecimento dos objetivos do estudo, as mães que aceitaram participar assinaram o Termo de Consentimento Livre e Esclarecido. 
O retorno às participantes do estudo foi feito a partir da entrega e explicação de um fôlder contendo orientações para uma alimentação saudável no pós-parto e do encaminhamento para atendimento nutricional das mulheres nas seguintes situações: que tinham sobrepeso ou obesidade pré-gestacional e que continuaram com sobrepeso até o sexto mês pós-parto; que estavam com obesidade nos primeiros seis meses pós-parto; e aquelas que possuíam sobrepeso e obesidade associados a outras doenças.

Ressalta-se também que, no momento da coleta de dados, foram sanadas as dúvidas por parte das voluntárias quanto a outros aspectos da nutrição materno-infantil. Por sua vez, o retorno dos resultados obtidos pela pesquisa para o setor de vacinação foi realizado pelo envio do relatório final do trabalho de conclusão de curso à coordenação do serviço e disponibilização do material educativo criado, para que a instituição possa utilizá-lo com outras mães que se enquadrarem no contexto.

Finalmente, vale ressaltar que o estudo não foi invasivo, sendo realizado em curto espaço de tempo e as mães receberam retorno quanto ao seu estado nutricional. Além disso, não foram negados esclarecimentos às mulheres que não aceitaram participar do estudo mas tiveram dúvidas no âmbito da retenção de peso pós-parto.

\section{Resultados}

Foram analisadas 34 mulheres ao todo, as quais, em sua maioria, eram naturais de Viçosa $(70,6 \%, n=24)$ e todas residentes nesta cidade. A média de idade encontrada foi 27,24 ( $\pm 6,9$ anos) e mediana de 27 anos, sendo a idade mínima 18 e a máxima, 42. Na tabela 1, constam os dados sociodemográficos, comportamentais e econômicos das participantes, e observa-se que a maioria das mulheres não trabalhava $(n=22 ; 64,7 \%)$, e dentre as que trabalhavam, 2,9\% $(n=1)$ estavam com período menor ou igual a quatro meses pós-parto e não estavam de licença-maternidade. 
Tabela 1. Características sociodemográficas, comportamentais e econômicas das participantes do estudo. Viçosa-MG, 2014.

Características
Frequência Absoluta

(n)

Idade

18-30

31-42

Estado Civil

Casada

União Estável

Solteira

Separada

Trabalha

Sim

Não

Licença-Maternidade

Sim

Não

Álcool

Sim

Não

Prática de Atividade Física

Sim

Não

Classificação Econômica*

B

C

D

Total

20

14

13

7

12

2

12

22

6

28

8

26

3

31

5

24

5

34
58,8

41,2

38,2

20,6

35,3

5,9

35,3

64,7

17,6

82,4

23,5

76,5

8,8

91,2

14,7

70,6

14,7

100

*Critério de Classificação Econômica Brasil - Associação Brasileira de Empresas de Pesquisa (ABEP) 
Como pode ser verificado na tabela 2 , em relação às características obstétricas e do pós-parto, houve maior frequência de mulheres com menos de seis meses pós-parto $(n=25 ; 73,5 \%)$. A média de idade gestacional foi de 39,2 ( $\pm 1,07)$, mediana de 39 semanas, sendo que a idade mínima foi 37 e máxima de 42 semanas respectivamente; por sua vez, a média do peso ao nascer foi $3.255 \mathrm{~g}$ $( \pm 393 \mathrm{~g})$ e a mediana $3.253 \mathrm{~g}$, havendo predomínio de crianças com peso normal ao nascer $(\mathrm{n}=$ 24; 70,6\%). Entre as multíparas, o intervalo entre as gestações teve uma média de 6,22 ( $\pm 3,49$ anos) e mediana de sete anos.

Tabela 2. Características obstétricas e do período pós-parto. Viçosa-MG, 2014.

\begin{tabular}{lcc}
\hline Características & $\begin{array}{c}\text { Frequência Absoluta } \\
(\mathrm{n})\end{array}$ & $\begin{array}{c}\text { Frequência Relativa } \\
(\%)\end{array}$ \\
\hline $\begin{array}{l}\text { Idade Pós-Parto } \\
\leq 6 \text { meses }\end{array}$ & 25 & 73,5 \\
$>6$ meses & 9 & 26,5 \\
Peso ao nascer & & \\
Macrossomia & 2 & 5,9 \\
Normal & 24 & 70,6 \\
Peso Insuficiente & 8 & 23,5 \\
Paridade & & \\
Primípara & 18 & 52,9 \\
Multípara & 16 & 47,1 \\
Total & 34 & 100 \\
\hline
\end{tabular}

De acordo com o que se verifica na tabela 3, quando questionadas sobre o aleitamento materno, $84 \%$ ( $n=21)$ das mães com idade pós-parto menor ou igual a seis meses e 55,6\% (n=5) das mães com período maior que seis meses pós-parto responderam que estavam amamentando, sendo que 4,0\% $(n=1)$ das mães com menos de seis meses pós-parto ofereciam leite materno e complementavam com algum tipo de fórmula láctea; além disso, de todas as mulheres que não amamentavam atualmente $(n=8 ; 23,5 \%)$, apenas uma nunca ofereceu leite materno. 
Tabela 3. Ocorrência da amamentação de acordo com o período pós-parto. Viçosa-MG, 2014.

\begin{tabular}{lcccc}
\hline \multirow{2}{*}{ Amamenta } & \multicolumn{2}{c}{ Idade pós- parto } & Total \\
\cline { 2 - 4 } Sim & 21 & $>6$ meses & 26 \\
\multirow{2}{*}{ Não } & 4 & 5 & 8 \\
\hline \multicolumn{2}{c}{ Total } & 25 & 4 & 34 \\
\hline
\end{tabular}

O percentual de retenção de peso foi maior que o de não retenção nos dois períodos analisados, mas é preocupante observar que as prevalências de retenção de peso em mulheres com idade pós-parto maior que seis meses e naquelas com idade menor ou igual a seis meses foram valores próximos, conforme é apresentado na figura 1.

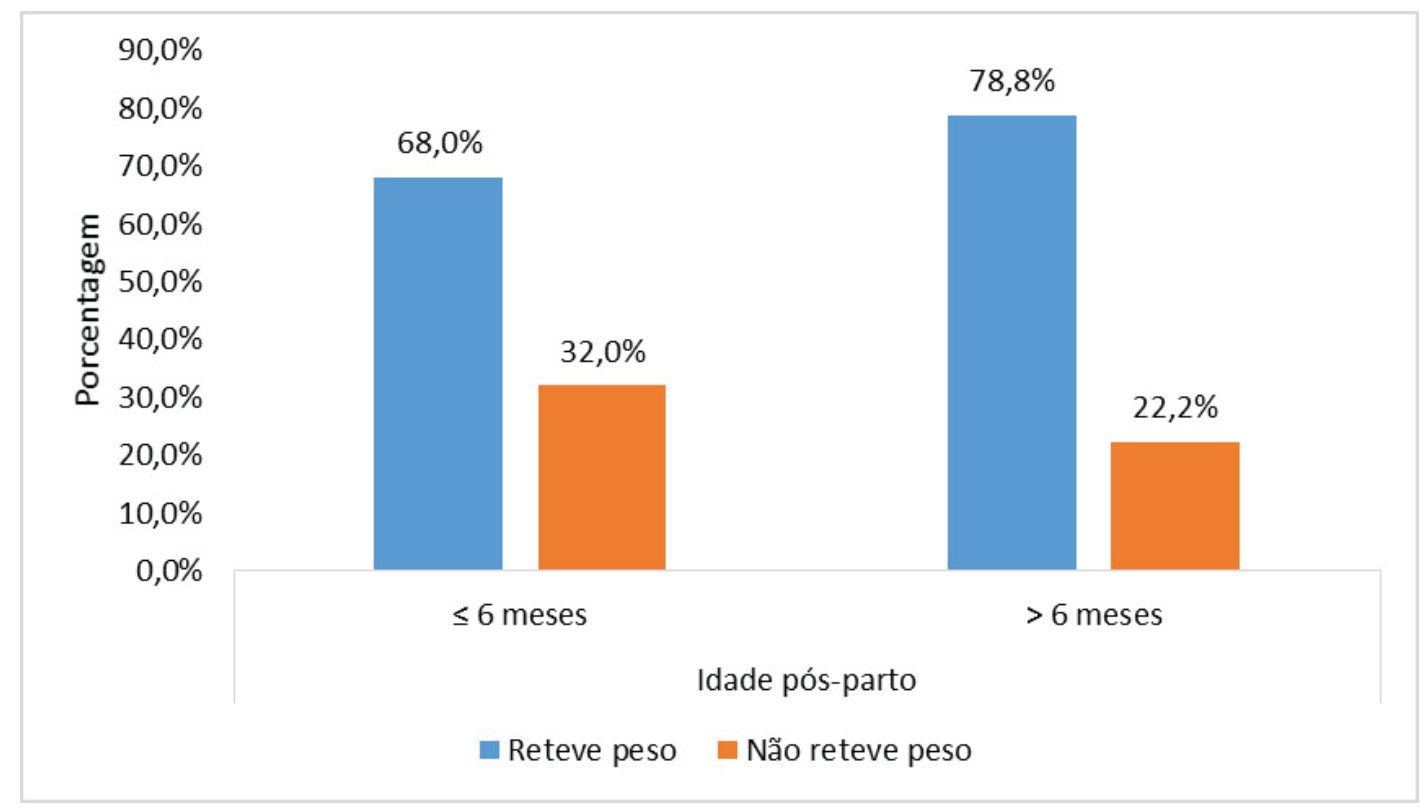

Figura 1. Distribuição da retenção de peso de acordo com a idade pós-parto. Viçosa-MG, 2014. 
Ainda vale ressaltar que, de todas as mulheres que retiveram peso no período pós-parto, $16,7 \%(n=4)$ eram obesas, $54,2 \%(n=13)$ tinham sobrepeso e o restante 29,1\% ( $n=7)$, eutróficas. Comparando-se o peso ganho na gestação e o peso retido, verificou-se que $5,9 \%(\mathrm{n}=2)$ de todas as participantes retiveram peso maior do que o adquirido no período gestacional - ou seja, além de não perderem o peso ganho, tiveram um incremento ponderal, sendo que tinham mais que seis meses pós-parto.

De acordo com as faixas de ganho de peso recomendados para gestantes de acordo com o estado pré-gestacional determinados pelo Institute of Medicine, ${ }^{13}$ a mesma quantidade de mulheres deste estudo ganhou peso acima $(\mathrm{n}=12 ; 35,3 \%)$ e dentro do adequado $(\mathrm{n}=12 ; 35,3 \%)$ conforme o peso pré-gestacional.

Segundo a figura 2, pode-se notar que, em ambos períodos analisados, houve maior percentual de eutrofia no período pré-gestacional em comparação ao IMC atual. Além disso, verifica-se elevada prevalência de sobrepeso quanto ao IMC atual no período menor ou igual a seis meses pós-parto.

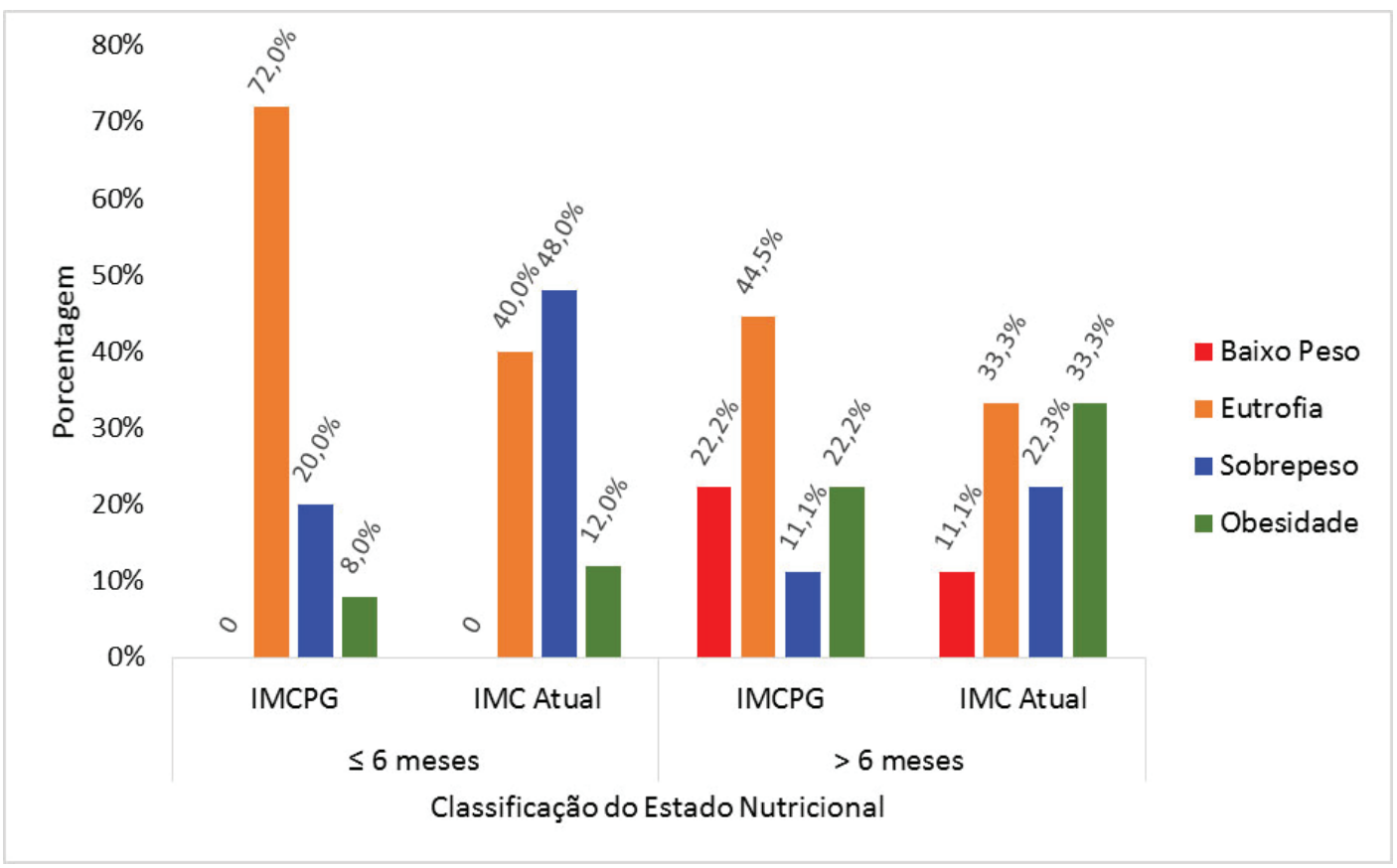

Figura 2. Classificação das mães quanto ao estado nutricional pré-gestacional e atual de acordo com o período pós-parto. Viçosa-MG, 2014. 
A tabela 4 representa a distribuição da média de retenção de peso pós-parto de acordo com características sócio demográficas, comportamentais, econômica e obstétricas, e pode-se observar que não houve diferença significativa da retenção de peso entre as idades maternas analisadas, quanto ao hábito de ingerir bebida alcoólica, à prática de atividade física, à condição socioeconômica, nem quanto a paridade e peso da criança ao nascer.

Tabela 4. Média e desvio-padrão da retenção de peso pós-parto segundo características comportamentais, econômica e obstétricas. Viçosa-MG, 2014.

\begin{tabular}{|c|c|c|c|c|}
\hline Características & $\mathrm{n}$ & $\begin{array}{l}\text { Média de retenção } \\
\text { de peso pós-parto }\end{array}$ & $\begin{array}{l}\text { Desvio } \\
\text { Padrão }\end{array}$ & $\mathrm{p}^{*}$ \\
\hline \multicolumn{5}{|l|}{ Idade } \\
\hline $18-30$ & 20 & 3,14 & 6,00 & 0,433 \\
\hline $31-42$ & 14 & 4,84 & 6,32 & \\
\hline \multicolumn{5}{|l|}{ Álcool } \\
\hline Sim & 8 & 7,64 & 5,95 & 0,042 \\
\hline Não & 26 & 2,67 & 5,76 & \\
\hline \multicolumn{5}{|l|}{ Atividade Física } \\
\hline Sim & 3 & 1,57 & 7,3 & 0,508 \\
\hline Não & 31 & 4,06 & 6,07 & \\
\hline \multicolumn{5}{|c|}{ Classificação Econômica } \\
\hline $\mathrm{B}$ & 5 & 3,66 & 6,24 & \\
\hline $\mathrm{C}$ & 24 & 2,87 & 5,90 & 0,156 \\
\hline $\mathrm{D}$ & 5 & 8,66 & 5,75 & \\
\hline \multicolumn{5}{|l|}{ Peso ao nascer } \\
\hline Macrossomia & 2 & 3,35 & 2,62 & \\
\hline Normal & 24 & 4,40 & 6,74 & 0,703 \\
\hline Peso Insuficiente & 8 & 2,28 & 4,58 & \\
\hline \multicolumn{5}{|l|}{ Paridade } \\
\hline Primípara & 18 & 3,75 & 6,52 & 0,930 \\
\hline Multípara & 18 & 3,94 & 5,79 & \\
\hline \multicolumn{5}{|c|}{ Ganho de peso gestacional } \\
\hline Excessivo & 12 & 7,98 & 6,60 & \\
\hline Insuficiente & 10 & 0,44 & 5,47 & 0,006 \\
\hline Adequado & 12 & 2,53 & 3,52 & \\
\hline Total & 34 & & & \\
\hline
\end{tabular}

*teste $t$ Student (comparações entre duas categorias) ou ANOVA (comparações entre três categorias) 
No entanto, houve diferença significativa da retenção de peso quanto ao ganho de peso gestacional $(\mathrm{p}=0,006)$, sendo que as que ganharam peso excessivo durante a gestação, de acordo com o estado nutricional pré-gestacional, apresentaram maior retenção de peso $(7,98 \mathrm{~kg} \pm 6,6)$ em relação àquelas que ganharam peso insuficiente ou adequado.

Conforme o teste de Tukey, realizado para determinar a magnitude das diferenças entre as médias de retenção de peso quanto a variável ganho de peso gestacional, observou-se que houve diferença significativa na retenção de peso das mulheres que ganharam peso excessivo na gestação em relação àquelas que ganharam peso insuficiente; porém, não houve diferença significativa entre aquelas com ganho de peso insuficiente e com ganho de peso adequado.

É importante mencionar também que houve algumas limitações neste estudo, tais como: o autorrelato do peso pré-gestacional e do peso adquirido durante a gestação podem ter sido sub ou superestimados; a amostra reduzida também pode ter limitado o estudo pois impossibilitou a realização de mais testes estatísticos e a possível verificação da associação de outras variáveis com a retenção de peso.

No entanto, a associação verificada neste estudo (retenção de peso e ganho de peso gestacional) também foi encontrada em outros estudos ${ }^{7,9,14,15-18}$ com amostras maiores, o que sugere que tal resultado poderia ter sido encontrado se a amostra fosse maior.

\section{Discussão}

Os resultados deste estudo revelam maior percentual de mulheres com retenção de peso no período maior que seis meses pós-parto, o que é preocupante, pois espera-se que a mulher nesse período já tenha perdido a reserva de peso adquirido na gestação. Essa perda ocorre principalmente nos primeiros três meses após o parto, mantendo-se em ritmo mais lento e constante até aproximadamente o sexto mês. ${ }^{1}$ Observou-se valor relativamente alto de mães com sobrepeso no período menor ou igual a seis meses pós-parto e com obesidade no período maior que seis meses pós parto.

O sobrepeso verificado no período pós-parto menor ou igual a seis meses é esperado, pois representa uma reserva de gordura da mulher, e é considerado fator positivo do ponto de vista da amamentação. Desde o início da gestação, o organismo se prepara para a produção de leite, e o tecido adiposo acumulado funciona como reserva energética para a produção de leite materno nos primeiros meses pós-parto. ${ }^{1}$ Entretanto, o sobrepeso e obesidade encontrados em mulheres com idade pós-parto maior que seis meses representa uma preocupação, pois pode predizer existência de gordura corporal além da necessária para suprir a demanda energética aumentada da nutriz. Esse depósito excessivo de tecido adiposo pode não ser utilizado no pós-parto, possibilitando a retenção de peso, colaborando assim com o aumento da prevalência de mulheres obesas. ${ }^{2}$ 
Houve maior retenção de peso entre as mulheres que tiveram ganho ponderal excessivo durante a gestação em detrimento daquelas com ganho de peso adequado ou insuficiente. Tal achado é semelhante ao que Rebelo et al., ${ }^{7}$ Nast et al. ${ }^{14}$ e Vasconcelos et al. ${ }^{15}$ encontraram em seus estudos realizados no período de um a um mês e meio pós-parto e de seis semanas a seis meses pós-parto, respectivamente.

O ganho ponderal durante a gestação tem sido apontado como importante determinante para a retenção de peso no pós-parto, uma vez que quanto maior o ganho de peso gestacional, maior a retenção de peso no pós-parto. ${ }^{5,16,17}$ Neste sentido, estudos citados por Zanotti ${ }^{9}$ apontam que mulheres que apresentam elevado ganho ponderal durante a gestação são propensas a reter mais peso, independentemente da classificação de índice de massa corporal (IMC) pré-gestacional, colaborando para a retenção de peso no período pós-parto.

O Institute of Medicine ${ }^{13}$ recomenda faixas de ganho de peso durante a gestação de acordo com o IMC pré-gestacional, porém essas recomendações têm por objetivo promover o bom crescimento fetal. No entanto, não se conhecem os efeitos dessas recomendações na retenção de peso ao logo da vida da mulher; assim, o ganho de peso acima das recomendações pode constituir importante fator para a retenção de peso., ${ }^{1,2,16}$

Ainda em relação à idade materna, o estudo de coorte realizado por Kac et al. ${ }^{4}$ sobre a evolução da retenção de peso em mulheres, ao longo de nove meses pós-parto, constatou que as mulheres menores de 20 anos de idade apresentaram menor retenção de peso em relação as demais.

Corroborando o resultado encontrado no presente estudo quanto à paridade, mulheres primíparas tendem a ganhar mais peso durante a gravidez em relação a multíparas., ${ }^{2,16,17}$ No entanto, Castro, Kac \& Sichieri ${ }^{2}$ encontraram em seu estudo que mulheres mais velhas possuem menor tendência em ganharem peso elevado. As variáveis "idade materna", "paridade", "renda" e "escolaridade" estão relacionadas à modificação de peso no pós-parto. ${ }^{1,2}$

A escolaridade foi avaliada juntamente com a situação econômica no critério de classificação econômica utilizado, portanto, subentende-se que mães que foram classificadas com classe econômica inferior possuem também menor nível de escolaridade. Neste sentido, considera-se que no grupo estudado houve maior retenção de peso entre mães com menor poder aquisitivo e baixo nível de escolaridade, o que vai ao encontro aos estudos conduzidos por Kac et al., ${ }^{4}$ Colebrusco $^{8} \mathrm{e}$ Zanoti, Cap e Wender, ${ }^{18}$ os quais observaram que a retenção de peso se reduz conforme o aumento do nível de escolaridade.

Não foi observada diferença significativa na retenção de peso entre as participantes sedentárias e aquelas praticavam atividade física. No entanto, Castro, Kac e Sichieri, ${ }^{2}$ em sua revisão de literatura, citam estudos que apontam que a atividade física praticada regularmente por mulheres no pós-parto auxilia no retorno ao peso pré-gestacional e ajuda a promover menor retenção do peso adquirido 
na gestação. Segundo Leitão et al. ${ }^{19}$ e Kac et al., ${ }^{20}$ entre os objetivos da prática de atividade física por gestantes estão o melhor controle do peso e a recuperação mais rápida no pós-parto imediato.

\section{Conclusão}

Embora o pequeno número amostral tenha limitado a realização de algumas associações estatísticas, observou-se relação entre retenção de peso pós-parto e ganho de peso gestacional, sendo que as mulheres que ganharam peso excessivo durante a gestação tiveram maior retenção de peso do que aquelas que ganharam peso insuficiente ou adequado.

Como ponto positivo, pode ser mencionada a elevada prevalência de aleitamento materno entre as mães estudadas.

Ressalta-se a necessidade da realização de mais estudos sobre retenção de peso pós-parto, uma vez que as modificações de peso ocorridas durante as fases de gestação e pós-parto estão intimamente associadas ao desenvolvimento da obesidade com o passar dos anos.

Diante disso, o presente estudo analisou uma ampla faixa de idade pós-parto (do $2^{\circ}$ ao $18^{\circ}$ mês pós-parto), que foram agrupadas em dois períodos, sendo mulheres com idade menor ou igual a seis meses pós-parto e mulheres com mais de seis meses pós-parto, e embora tenha sido uma pequena amostra, foi possível analisar em qual período houve maior prevalência das variáveis analisadas.

\section{Referências}

1. Lacerda EMA, Leal MC. Fatores associados com a retenção e o ganho de peso pós-parto: uma revisão sistemática. Rev. Bras. Epidemiol. 2004; 7(2):187-200.

2. Castro MBT, Kac G, Sichieri R. Determinantes nutricionais e sóciodemográficos da variação de peso no pós-parto: uma revisão da literatura. Rev. Bras. Saúde Mater. Infant. 2009; 9(2):125-137.

3. Instituto Brasileiro de Geografia e Estatística. Pesquisa de orçamentos familiares 2008-2009: antropometria e estado nutricional de crianças, adolescentes e adultos no Brasil. Rio de Janeiro: IBGE; 2010. 130 p.

4. Kac G, D’Aquino BMH, Valente JG, Velásquez-Meléndez G. Post partum weight retention among women in Rio de Janeiro: a follow-up study. Cad. Saúde Pública 2003; 19(Sup. 1):S149-S161.

5. Lacerda EMA, Saunders C. Nutrição da nutriz. In: Accioly E, Saunders C, Lacerda EMA. Nutrição em obstetrícia e pediatria. 2.ed. Rio de Janeiro: Cultura Médica: Guanabara Koogan; 2009. p. 231-237.

6. Brasil. Ministério da Saúde. Centro Brasileiro de Análise e Planejamento. Pesquisa Nacional de Demografia e Saúde da Mulher e da Criança - PNDS 2006: Relatório Final. Brasília: CEBRAP; 2008. 583 p. 
7. Rebelo F, Castro MBT, Dutra CL, Schlussel MM, Kac G. Fatores associados à retenção de peso pósparto em uma coorte de mulheres, 2005-2007. Rev. Bras. Saúde Mater. Infant. 2010; 10(2):219-227.

8. Colebrusco LDO. Fatores determinantes da variação de peso no período pós-parto [dissertação]. São Paulo: Faculdade de Saúde Pública, Universidade de São Paulo; 2010.

9. Zanotti J. Fatores relacionados à retenção de peso no pós-parto [dissertação]. Porto Alegre: Faculdade de Medicina, Universidade Federal do Rio Grande do Sul; 2012.

10. Organización Mundial de La Salud. El estado físico: uso e interpretación de La antropometría: informe de un comité de expertos de la OMS. Genebra: OMS; 1999. 513 p.

11. World Health Organization. Physical status: the use and interpretation of antropometry. Geneva: WHO; 1995.

12. World Health Organization. Obesity status: preventing and managing the global epidemic. Report of a WHO consultation on obesity. Geneve: WHO; 1998.

13. Institute of Medicine. Weight gain during pregnancy: reexamining the guidelines. Washington DC: National Academies Press; 2009.

14. Nast M, Oliveira A, Rauber F, Vitolo MR. Ganho de peso excessivo na gestação é fator de risco para o excesso de peso em mulheres. Rev. Bras. Ginecol. Obstet. 2013; 35(12):536-40.

15. Vasconcelos C, Costa FC, Almeida PC, Araujo Jr E, Sampaio HAC. Fatores de risco associados à retenção de peso seis meses após o parto. Rev. Bras. Ginecol. Obstet. 2014; 36(5):222-7.

16. Kac G. Fatores determinantes da retenção de peso no pós-parto: uma revisão da literatura. Cad. Saúde Pública 2001; 17(3):455-466.

17. Konno SC, Benicio MHD, Barros AJD. Fatores associados à evolução ponderal de gestantes: uma análise multinível. Rev. Saúde Pública 2007; 41(6):995-1002.

18. Zanotti J, Capp E, Wender MCO. Factors associated with postpartum weight retention in a Brazilian cohort. Rev. Bras. Ginecol. Obstet. 2015; 37(4):164-171.

19. Leitão MB, Lazzoli JK, Oliveira MAB, Nóbrega ACL, Silveira GG, Carvalho T, et al. Posicionamento oficial da Sociedade Brasileira de Medicina do Esporte: atividade física e saúde na mulher. Rev. Bras. Med. Esporte 2000; 6(6):215-220.

20. Kac G, Pacheco AHRN, Araújo DMR, Rocha CMM, Sousa EB, Coelho NLP, et al. Fatores associados à prática de atividade física no lazer em mulheres no pós-parto. Rev. Bras. Med. Esporte 2007; 13(2):86-90. 
